\title{
Optimum Solutions of Fredholm and Volterra Integro-differential Equations
}

\author{
Muhammad Akbar, Rashid Nawaz, Sumbal Ahsan \\ Department of Mathematics, Abdul Wali Khan University, Mardan, Pakistan \\ Email address: \\ aimakbar143bj@gmail.com (M. Akbar),rashid_uop@yahoo.com (R. Nawaz), sumbalahsan99@gmail.com(S. Ahsan)

\section{To cite this article:} \\ Muhammad Akbar, Rashid Nawaz, Sumbal Ahsan. Optimum Solutions of Fredholm and Volterra Integro-differential Equations. \\ International Journal of Theoretical and Applied Mathematics. Vol. 5, No. 6, 2019, pp. 100-112. doi: 10.11648/j.ijtam.20190506.14
}

Received: April 5, 2019; Accepted: November 29, 2019; Published: December 6, 2019

\begin{abstract}
Integro-differential equations arise in modeling various physical and engineering problems. Several numerical and analytical methods have been developed for solving integro-differential equations. In this paper, a powerful semi analytical technique known as Optimal Homotopy Asymptotic Method (OHAM) has been used for finding the approximate solutions of Fredholm type integro-differential equations and Volterra type integro-differential equations. The proposed method does not required discretization like other numerical and approximate method, and it is also free from any small/large parameters. The presented technique provides better accuracy at lower order of approximation, the accuracy of the method can further be increases with higher order of approximation. Moreover, we can easily adjust and control the convergence region. The ability of the method is checked with different problems in literature. The results obtained through OHAM are compared with solutions of Adomian Decomposition Method. It is observed that solutions obtained through the proposed method is more accurate than existing techniques, which proves the validity and stability of the proposed method for solving integrodifferential equations. The presented technique is more consistent, effective, suitable and rapidly convergent. The use of Optimal Homotopy Asymptotic Method is simple and straight forward. For the computation of problems, we have used Mathematica 9.0.
\end{abstract}

Keywords: Integro-differential Equations, Approximate Solutions, Optimal Homotopy Asymptotic Method

\section{Introduction}

Numerous mathematical formulations of physical phenomena involve integro-differential equations (IDE), these equations arise in many fields of science like chemical kinetics, fluid dynamics and biological models. In fact, obtaining the exact solutions of integro-differential equations are usually problematic so it is mandatory to attain efficient approximate solutions. Different approaches in literature have been used for the solutions of these equations. Some of them are Variational Iterative Method (VIM) [1-2], Modified Homotopy Perturbation Method (MHPM) [3-4], Homotopy Analysis Transform Method (HAM) [5-8], Conjugate Gradient Method (CGM) [9], Adomian Decomposition Method (ADM) [10-11] and Block Pulse Functions (BPF) [12] etc. Optimal Homotopy Asymptotic method (OHAM) is one of the most power full techniques for finding the approximate solutions of differential and integro differential equations. The method was introduced by Marinca et al. [13,
14] for the solution of differential equations. Many authors extended the proposed method for the solution of different kinds of integral equations. Khan et al. [15] used the proposed method for the solution of Volterra integral equation of the first kind. Almousa et al. [16] applied it for the solution of linear Fredholm integral equations of the first kind. Hashmi et al. [17] implemented the proposed method for the solution of Fredholm integro-differential equations.

In this article, we apply the proposed method for finding the approximate solutions of Volterra and Fredholm integrodifferential equations. The general nth order integrodifferential equations [18] has the following form

$$
\psi^{n}(\mathrm{~s})+\sum_{i=0}^{n-1} \psi^{i}(\mathrm{~s}) f_{i}(s)+\int_{a}^{b} k(s, t) \psi^{m}(t) d t=g(\mathrm{~s}), \quad \mathrm{a}<\mathrm{s}<\mathrm{b},
$$

with initial conditions 


$$
\psi(\mathrm{a})=\alpha_{0}, \psi^{\prime}(\mathrm{a})=\alpha_{1}, \psi^{\prime \prime}(\mathrm{a})=\alpha_{2}, \ldots . \psi^{(\mathrm{n}-1)}(\mathrm{a})=\alpha_{n-1},
$$

where $\alpha_{i}{ }^{\prime} s$ are real constants, $m$ and $n$ are integers such that $m<n, f_{i}^{\prime} s, g$ and $k$ are given functions and $\psi$ is the solution to be determined. The error analysis of the numerical problems confirms convergence and stability of the proposed method.

This paper is divided into five main sections. In section 2 , basic idea of OHAM is introduced. Section 3 consists of numerical problems. Section 4 is the results and discussion, while in section 5 some conclusions are drawn.

\section{Basic Idea of OHAM}

In this section, the formulation of OHAM is presented, consider equation of the form:

$$
L(\psi(\mathrm{s}))+\mathrm{g}(\mathrm{s})+N(\psi(\mathrm{s}))=0, \mathrm{~B}\left(\psi, \frac{d \psi}{d s}\right)=0 .
$$

where $L$ is for linear and $N$ for non-linear operator, $\psi(\mathrm{s})$ is unknown function and $g(\mathrm{~s})$ is known function while $B$ is boundary operator. We obtain the family of equations by introducing an embedding parameter $p \in[0,1]$ as follows:

$$
\begin{gathered}
(1-p)\{L(\psi(s, p))+g(s)\}=H(p)\{L(\psi(s, p))+g(s)+N(\psi(s, p))\} \\
B\left(\psi(s, p), \frac{d \psi(\mathrm{s}, p)}{d s}\right)=0 .
\end{gathered}
$$

where $\psi(s, p)$ is unknown function, and for $p \neq 0$ the nonzero auxiliary function $H(p)$ is given as

$$
\begin{aligned}
& H(p)=c_{1} p^{1}+c_{2} p^{2}+c_{3} p^{3}+\ldots . .+c_{m} p^{m}, \\
& L\left(\psi_{k}(\mathrm{~s})-\psi_{k-1}(\mathrm{~s})\right)=c_{k} N_{0}\left(\psi_{0}(\mathrm{~s})\right)+\sum_{i=1}^{k-1} c_{i}\left[\begin{array}{l}
L\left(\psi_{k-i}(\mathrm{~s})\right) \\
+N_{k-i}\left(\psi_{0}(\mathrm{~s}), \psi_{1}(\mathrm{~s}), \ldots, \psi_{k-1}(\mathrm{~s})\right)
\end{array}\right], B\left(\psi_{k}, \frac{d \psi_{k}}{d s}\right)=0, \mathrm{k}=2,3, \ldots, \\
& N\left(\psi\left(\mathrm{s}, \mathrm{p} ; \mathrm{c}_{i}\right)\right)=N_{0}\left(\psi_{0}(\mathrm{~s})\right)+\sum_{k=1}^{\infty} N_{k}\left(\psi_{0}, \psi_{1}, \ldots, \psi_{k}\right) p^{k}
\end{aligned}
$$

where $\psi\left(\mathrm{s}, \mathrm{p} ; \mathrm{c}_{i}\right)$ is given by Eq. (6) It should be noted that $\psi_{k}$ for $k \geq 0$ is given by the Eq. (5) and Eq. (8) with the boundary conditions that come from original problem, which can be solved easily. If the series (6) convergent at $p=1$, then we have where $c_{1}, c_{2}, c_{3}, \ldots ., c_{m}$ are auxiliary constants and for $p=0$, $H(p)=0$. When $p=0$ or $p=1$ clearly, we have

$$
\begin{array}{r}
p=0 \Rightarrow H(\psi(\mathrm{s}, 0), 0)=L(\psi(\mathrm{s}, 0))+g(\mathrm{~s})=0, \\
p=1 \Rightarrow H(\psi(\mathrm{s}, 1), 1)=H(1)\{L(\psi(\mathrm{s}, p))+g(\mathrm{~s})+N(\psi(\mathrm{s}, p))\}=0 .
\end{array}
$$

Obviously when $p=0$ and $p=1$. It keeps that $\psi(s, 0)=\psi_{0}(s)$, and $\psi(s, 1)=\psi(s)$, respectively. So as $p$ varies from 0 to 1 , the result $\psi(\mathrm{s}, p)$ approaches from $\psi_{0}(\mathrm{~s})$ to $\psi(\mathrm{s})$, where $\psi_{0}(\mathrm{~s})$ is obtained Eq (2). for $p=0$ :

$$
L\left(\psi_{0}(\mathrm{~s})\right)+g(\mathrm{~s})=0, \beta\left(\psi_{0}, \frac{d \psi_{0}}{d s}\right)=0 .
$$

The Taylor's series expansion about $p$ for obtaining the approximate solution, we write as follows:

$$
\psi\left(s, p ; c_{i}\right)=\psi_{0}(s)+\sum_{\kappa=1}^{\infty} \psi_{\kappa}\left(s, c_{i}\right) p^{\kappa}, \quad i=1,2, \ldots
$$

when $p=1$, one has written the series (6) as

$$
\psi\left(s, 1 ; c_{i}\right)=\psi_{0}(s)+\sum_{\kappa=1}^{\infty} \psi_{\kappa}\left(s, c_{i}\right), \quad i=1,2, \ldots
$$

when Eq. (6) is substituted in Eq. (2) and the coefficients of like power of $p$ is compare, we obtain the equation of $\psi_{0}(\mathrm{~s})$ given by (5) and the governing equations of $u_{k}(\mathrm{~s})$ is obtained as follows:

$$
L\left(\psi_{1}(\mathrm{~s})\right)=c_{1} N_{0}\left(\psi_{0}(\mathrm{~s})\right), \quad B\left(\psi_{1}, \frac{d \psi_{1}}{d s}\right)=0,
$$

$$
\psi\left(\mathrm{s}, \mathrm{c}_{i}\right)=\psi_{0}(\mathrm{~s})+\sum_{k=1}^{\infty} \psi_{k}\left(\mathrm{~s}, \mathrm{c}_{i}\right)
$$

The solution of Eq. (1) can be expressed approximately in the form:

$$
\psi^{m}\left(\mathrm{~s}, \mathrm{c}_{i}\right)=\psi_{0}(\mathrm{~s})+\sum_{k=1}^{\infty} \psi_{k}\left(\mathrm{~s}, \mathrm{c}_{i}\right), \quad i=1,2,3 \ldots, m .
$$

As the solution contains the auxiliary constants $c_{i}, i=1,2, \ldots$. we form the residual equation by putting Eq. (10) into Eq. (1) to find these constants as follows: 
$R\left(\mathrm{~s}, \mathrm{c}_{i}\right)=\mathrm{L}\left(\psi^{m}\left(\mathrm{~s}, \mathrm{c}_{i}\right)\right)+g(\mathrm{~s})+N\left(\psi^{m}\left(\mathrm{~s}, \mathrm{c}_{i}\right)\right), \quad i=1,2, \ldots, m$.

By using the least square method, we find $c_{i}, i=1,2, \ldots$. as

$$
J\left(c_{i}\right)=\int_{a}^{b} R^{2}\left(s, c_{i}\right) d s .
$$

Where $a$ and $b$ are the domain of the given problem. The constants $c_{i}, i=1,2,3, \ldots, m$ can be optimally identified from the conditions

$$
\begin{aligned}
\frac{\partial J}{\partial c_{1}}=\frac{\partial J}{\partial c_{2}}=\frac{\partial J}{\partial c_{2}}=\ldots=\frac{\partial J}{\partial c_{m}} & =0 . \\
u^{\prime \prime}(x)=x-\sin (x)-\int_{0}^{\frac{\pi}{2}} x t u(t) d t, & u(0)=0, \quad u^{\prime}(0)=1,
\end{aligned}
$$

$\mathrm{m})$ in eq. (10) is well determined.

\section{Implementation of OHAM to Integro-differential Equations}

In this section, the effectiveness and accuracy of OHAM is tested by solving Volterra and Fredholm integro-differential equations

Problem 1

Consider the Fredhohm integro-differential equation [19] of the following form

Eq. (14) has exact solution $u(x)=\sin (x)$. From the given equation

$$
\begin{aligned}
& L(u(x ; q))=u^{\prime \prime}(x), \\
& N(u(x ; q))=\int_{0}^{\frac{\pi}{2}} t x u(t) d t, \\
& f(x)=-(x-\sin (x)) .
\end{aligned}
$$

The homotopy using OHAM is as follow

$$
\begin{gathered}
(1-q)\left(\left(u_{0}{ }^{\prime \prime}(x)+q u_{1}{ }^{\prime \prime}(x)+q^{2} u_{2}{ }^{\prime \prime}(x)+\cdots\right)-(x-\sin (x))\right) \\
=H(q)\left(\begin{array}{c}
\left(u_{0}{ }^{\prime \prime}(x)+q u_{1}{ }^{\prime \prime}(x)+q^{2} u_{2}{ }^{\prime \prime}(x)+\cdots\right)-(x-\sin (x)) \\
+\int_{0}^{\frac{\pi}{2}} t x\left(u_{0}(t)+q u_{1}(t)+q^{2} u_{2}(t)+\cdots\right) d t
\end{array}\right), \\
u(0 ; q)=0, u^{\prime}(0 ; q)=1
\end{gathered}
$$

A series of problems is created by comparing the co-efficient of same power of $q$, the series is

$$
\begin{aligned}
& O\left(q^{0}\right): u_{0}{ }^{\prime \prime}(x)=x-\sin (x), \quad u_{0}(0)=0, \quad u_{0}^{\prime}(0)=1, \\
& O\left(q^{1}\right): u_{1}^{\prime \prime}(x)=c_{1} \int_{0}^{\frac{\pi}{2}} t x u_{0}(x) d t, \quad u_{1}(0)=0, \quad u_{1}^{\prime}(0)=0, \\
& O\left(q^{2}\right): u_{2}^{\prime \prime}(x)=\left(1+c_{1}\right) u_{1}^{\prime \prime}(x)+c_{2} \int_{0}^{\frac{\pi}{2}} t x u_{0}(x) d t+c_{1} \int_{0}^{\frac{\pi}{2}} t x u_{1}(x) d t, \\
& u_{2}(0)=0, \quad u_{2}^{\prime}(0)=0, \\
& O\left(q^{3}\right): \quad u_{3}^{\prime \prime}(x)=\left(1+c_{1}\right) u_{2}{ }^{\prime \prime}(x)+c_{2} u_{1}{ }^{\prime \prime}(x)+c_{3} \int_{0}^{\frac{\pi}{2}} t x u_{0}(x) d t+c_{2} \int_{0}^{\frac{\pi}{2}} t x u_{1}(x) d t \\
& +c_{1} \int_{0}^{\frac{\pi}{2}} t x u_{2}(x) d t, u_{3}(0)=0, \quad u_{3}^{\prime}(0)=0 .
\end{aligned}
$$


Solving eq. (19-22) we have

$$
\begin{gathered}
u_{0}(x)=\frac{1}{6}\left(x^{3}+6 \sin (x)\right), \\
u_{1}(x)=\frac{c_{1}\left(960+\pi^{5}\right) x^{3}}{5760}, \\
u_{2}(x)=\frac{1}{5529600}\left(960+\pi^{5}\right)\left(960 c_{1}+960 c_{1}^{2}+960 c_{2}+c_{1}^{2} \pi^{5}\right) x^{3}, \\
u_{3}(x)=\frac{1}{5308416000}\left(960+\pi^{5}\right)\left(\begin{array}{l}
921600 c_{1}+1843200 c_{1}^{2}+921600 c_{1}^{3} \\
+921600 c_{2}+1843200 c_{1} c_{2}+921600 c_{3} \\
+1920 c_{1}^{2} \pi^{5}+1920 c_{1}^{3} \pi^{5}+1920 c_{1} c_{2} \pi^{5}+c_{1}^{3} \pi^{10}
\end{array}\right) x^{3} .
\end{gathered}
$$

The $3^{\text {rd }}$ order approximate solution by OHAM is $u=u_{0}+u_{1}+u_{2}+u_{3}$

$$
\begin{aligned}
u= & \frac{1}{6}\left(x^{3}+6 \sin (x)\right)+\frac{c_{1}\left(960+\pi^{5}\right) x^{3}}{5760}+\frac{1}{5529600}\left(960+\pi^{5}\right)\left(\begin{array}{l}
960 c_{1}+960 c_{1}^{2} \\
+960 c_{2}+c_{1}^{2} \pi^{5}
\end{array}\right) x^{3} \\
& +\frac{1}{5308416000}\left(960+\pi^{5}\right)\left(\begin{array}{l}
921600 c_{1}+1843200 c_{1}^{2}+921600 c_{1}^{3}+921600 c_{2} \\
+1843200 c_{1} c_{2}+921600 c_{3}+1920 c_{1}{ }^{2} \pi^{5}+1920 c_{1}^{3} \pi^{5} \\
+1920 c_{1} c_{2} \pi^{5}+c_{1}^{3} \pi^{10}
\end{array}\right) x^{3} .
\end{aligned}
$$

The values of constants $c_{1}=-0.89060667, c_{2}=-1.30668415$ and $c_{3}=-0.452018363$ are calculated using method of least squares.

With these constants the approximate solution (27) become

$$
u=-0.1666667 x^{3}+\frac{1}{6}\left(x^{3}+6 \sin (x)\right) .
$$

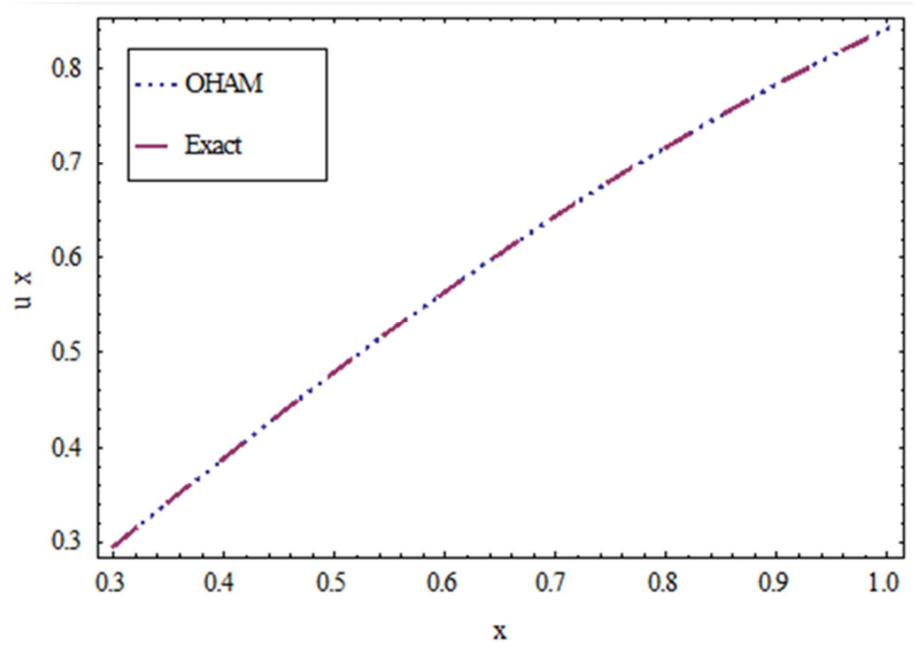

Figure 1. 2D plots of OHAM versus exact solution.

Table 1. Shows the comparison of $3^{\text {rd }}$ order OHAM solution with $5^{\text {th }}$ order ADM [19] and exact solution.

\begin{tabular}{lllll}
\hline $\mathbf{X}$ & Exact solutions & OHAM solutions & ADM absolute Errors & OHAM absolute Errors \\
\hline 0 & 0 & 0 & 0 & 0 \\
0.1 & 0.099833 & 0.099833 & $3.99167 \times 10^{-6}$ & 0 \\
0.2 & 0.198669 & 0.198669 & $3.19333 \times 10^{-5}$ & 0 \\
0.3 & 0.295520 & 0.295520 & $1.07775 \times 10^{-4}$ & $5.55112 \times 10^{-17}$ \\
\hline
\end{tabular}




\begin{tabular}{lllll}
\hline $\mathbf{X}$ & Exact solutions & OHAM solutions & ADM absolute Errors & OHAM absolute Errors \\
\hline 0.4 & 0.389418 & 0.389418 & $2.55467 \times 10^{-4}$ & 0 \\
0.5 & 0.479426 & 0.479426 & $4.98958 \times 10^{-4}$ & $5.55112 \times 10^{-17}$ \\
0.6 & 0.564642 & 0.564642 & $8.62200 \times 10^{-4}$ & 0 \\
0.7 & 0.644218 & 0.644218 & $1.36914 \times 10^{-3}$ & 0 \\
0.8 & 0.717356 & 0.717356 & $2.04373 \times 10^{-3}$ & 0 \\
0.9 & 0.783327 & 0.783327 & $2.90992 \times 10^{-3}$ & $1.11022 \times 10^{-16}$ \\
1 & 0.841471 & 0.841471 & $3.99167 \times 10^{-3}$ & $1.11022 \times 10^{-16}$ \\
\hline
\end{tabular}

Problem 2

Consider the following Fredhholm integro-differential equation [19]

$$
u^{\prime \prime}(x)=-\sin (x)+\cos (x)+\left(2-\frac{\pi}{2}\right) x-\int_{0}^{\frac{\pi}{2}} x t u(t) d t, \quad u(0)=-1, u^{\prime}(0)=1
$$

Eq. (29) has the exact solution $u(x)=\sin (x)-\cos (x)$. Using the Basic Idea of OHAM we have a series of problems

$$
\begin{gathered}
u_{0}^{\prime \prime}(x)=\left(2-\frac{\pi}{2}\right) x+\cos (x)-\sin (x), u_{0}(0)=-1, u_{0}^{\prime}(0)=1, \\
u_{1}^{\prime \prime}(x)=c_{1} \int_{0}^{\frac{\pi}{2}} t x u_{0}(t) d t, u_{1}(0)=0, u_{1}^{\prime}(0)=0, \\
u_{2}^{\prime \prime}(x)=\left(1+c_{1}\right) u_{1}^{\prime \prime}(x)+c_{2} \int_{0}^{\frac{\pi}{2}} t x u_{0}(t) d t+c_{1} \int_{0}^{\frac{\pi}{2}} t x u_{1}(t) d t, u_{2}(0)=0, u_{2}^{\prime}(0)=0, \\
u_{3}^{\prime \prime}(x)=\left(1+c_{1}\right) u_{2}^{\prime \prime}(x)+c_{2} u_{1}^{\prime \prime}(x)+c_{3} \int_{0}^{\frac{\pi}{2}} t x u_{0}(t) d t+c_{2} \int_{0}^{\frac{\pi}{2}} t x u_{1}(t) d t \\
+c_{1} \int_{0}^{\frac{\pi}{2}} t x u_{2}(t) d t, \quad u_{3}(0)=0, \quad u_{3}^{\prime}(0)=0 .
\end{gathered}
$$

Solving eq. $(30-33)$ we get

$$
\begin{gathered}
u_{0}(x)=\frac{1}{12}\left(4 x^{3}-\pi x^{3}-12 \cos (x)+12 \sin (x)\right), \\
u_{1}(x)=-\frac{1}{11520}\left(c_{1}\left(-3840+960 \pi-4 \pi^{5}+\pi^{6}\right) x^{3}\right), \\
u_{2}(x)=-\frac{1}{11059200}\left(960 c_{1}+960 c_{1}^{2}+960 c_{2}+c_{1}^{2} \pi^{5}\right)\left(-3840+960 \pi-4 \pi^{5}+\pi^{6}\right) x^{3}, \\
u_{3}(x)=-\frac{1}{10616832000}\left(-3840+960 \pi-4 \pi^{5}+\pi^{6}\right)\left(921600 c_{1}+1843200 c_{1}^{2}\right. \\
+921600 c_{1}^{3}+921600 c_{2}+1843200 c_{1} c_{2}+921600 c_{3}+1920 c_{1}^{2} \pi^{5}+1920 c_{1}^{3} \pi^{5} \\
\left.+1920 c_{1} c_{2} \pi^{5}+c_{1}^{3} \pi^{10}\right) x^{3} .
\end{gathered}
$$

The $3^{\text {rd }}$ order approximate solution by OHAM is $u=u_{0}+u_{1}+u_{2}+u_{3}$

$$
\begin{aligned}
u=\frac{1}{12}( & \left.4 x^{3}-\pi x^{3}-12 \cos (x)+12 \sin (x)\right)-\frac{1}{11520}\left(c_{1}\left(-3840+960 \pi-4 \pi^{5}+\pi^{6}\right) x^{3}\right) \\
& -\frac{1}{11059200}\left(960 c_{1}+960 c_{1}^{2}+960 c_{2}+c_{1}^{2} \pi^{5}\right)\left(-3840+960 \pi-4 \pi^{5}+\pi^{6}\right) x^{3} \\
& -\frac{1}{10616832000}\left(-3840+960 \pi-4 \pi^{5}+\pi^{6}\right)\left(921600 c_{1}+1843200 c_{1}^{2}\right. \\
& +921600 c_{1}^{3}+921600 c_{2}+1843200 c_{1} c_{2}+921600 c_{3}+1920 c_{1}^{2} \pi^{5}+1920 c_{1}^{3} \pi^{5} \\
& \left.+1920 c_{1} c_{2} \pi^{5}+c_{1}^{3} \pi^{10}\right) x^{3} .
\end{aligned}
$$


The value of constants $c_{1}=-0.89060667, c_{2}=-1.30668415$ and $c_{3}=-0.45201836$ are calculated using method of least squares.

With these constants the approximate solution (38) become

$$
u=-0.0715339 x^{3}+\frac{1}{12}\left(4 x^{3}-\pi x^{3}-12 \cos (x)+12 \sin (x)\right) .
$$

Table 2. Show the comparison of $3^{\text {rd }}$ order OHAM with $4^{\text {th }}$ order ADM [19] solution and exact solution.

\begin{tabular}{lllll}
\hline $\mathbf{X}$ & Exact solution & OHAM Solution & ADM absolute Error & OHAM absolute Errors \\
\hline 0 & -1 & -1 & 0 & 0 \\
0.1 & -0.895171 & -0.895171 & $7.38946 \times 10^{-7}$ & 0 \\
0.2 & -0.781397 & -0.781397 & $5.91156 \times 10^{-6}$ & 0 \\
0.3 & -0.659816 & -0.659816 & $1.99515 \times 10^{-5}$ & 0 \\
0.4 & -0.531643 & -0.531643 & $4.72925 \times 10^{-5}$ & 0 \\
0.5 & -0.398157 & -0.398157 & $9.23682 \times 10^{-5}$ & $5.55112 \times 10^{-17}$ \\
0.6 & -0.260693 & -0.260693 & $1.59612 \times 10^{-4}$ & $2.77556 \times 10^{-17}$ \\
0.7 & -0.120625 & -0.120625 & $2.53458 \times 10^{-4}$ & $1.38778 \times 10^{-17}$ \\
0.8 & 0.020649 & 0.020649 & $3.7834 \times 10^{-4}$ & $5.55112 \times 10^{-17}$ \\
0.9 & 0.161717 & 0.161717 & $5.38691 \times 10^{-4}$ & 0 \\
\hline
\end{tabular}

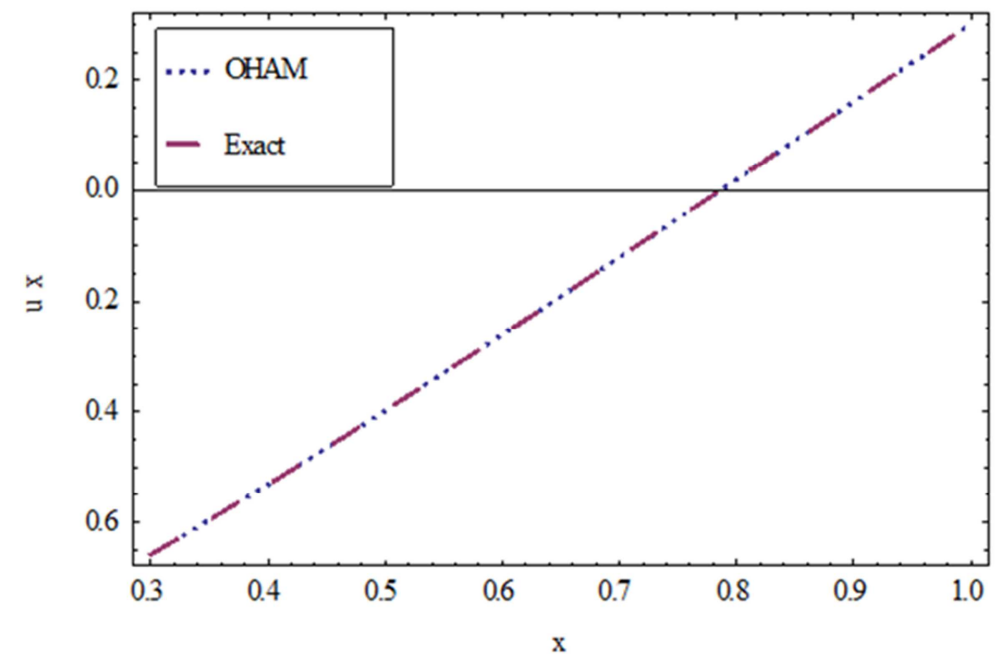

Figure 2. 2D plots of OHAM versus exact solution.

Problem 3

Taking the following Fredholm integro-differential equation [19]

$$
u^{\prime \prime}(x)=-e^{x}+\frac{x}{2}+\int_{0}^{1} x t u(t) d t, \quad u(0)=0, u^{\prime}(0)=-1,
$$

Eq. (40) has the exact solution $u(x)=1-e^{x}$. By using Basic idea of OHAM, we have a series of problems which are as follow

$$
\begin{gathered}
u_{0}{ }^{\prime \prime}(x)=e^{x}-\frac{x}{2}, \quad u_{0}(0)=0, u_{0}{ }^{\prime}(0)=-1, \\
u_{1}{ }^{\prime}(x)=-c_{1} \int_{0}^{1} t x u_{0}(t) d t, \quad u_{1}(0)=0, u_{1}^{\prime}(0)=0, \\
u_{2}^{\prime \prime}(x)=\left(1+c_{1}\right) u_{1}{ }^{\prime \prime}(x)-c_{2} \int_{0}^{1} t x u_{0}(t) d t-c_{1} \int_{0}^{1} t x u_{1}(t) d t, u_{2}(0)=0, u_{2}{ }^{\prime}(0)=0,
\end{gathered}
$$




$$
\begin{aligned}
u_{3}{ }^{\prime \prime}(x)= & \left(1+c_{1}\right) u_{2}{ }^{\prime \prime}(x)+c_{2} u_{1}{ }^{\prime \prime}(x)-c_{3} \int_{0}^{1} t x u_{0}(t) d t-c_{2} \int_{0}^{1} t x u_{1}(t) d t \\
& -c_{1} \int_{0}^{1} t x u_{2}(t) d t, \quad u_{3}(0)=0, u_{3}{ }^{\prime}(0)=0 .
\end{aligned}
$$

Solving Eq. $(41-44)$, we have

$$
\begin{gathered}
u_{0}(x)=\frac{1}{12}\left(12-12 e^{x}+x^{3}\right), \\
u_{1}(x)=\frac{29 c_{1} x^{3}}{360}, \\
u_{2}(x)=\frac{29}{10800}\left(30 c_{1} x^{3}+29 c_{1}^{2} x^{3}+30 c_{2} x^{3}\right), \\
u_{3}(x)=\frac{29}{324000}\left(900 c_{1} x^{3}+1740 c_{1}{ }^{2} x^{3}+841 c_{1}^{3} x^{3}+900 c_{2} x^{3}+1740 c_{1} c_{2} x^{3}+900 c_{3} x^{3}\right) .
\end{gathered}
$$

The $3^{\text {rd }}$ order approximate solution by OHAM is $u=u_{0}+u_{1}+u_{2}+u_{3}$

$$
\begin{aligned}
u= & \frac{1}{12}\left(12-12 e^{x}+x^{3}\right)+\frac{29 c_{1} x^{3}}{360}+\frac{29}{10800}\left(30 c_{1} x^{3}+29 c_{1}^{2} x^{3}+30 c_{2} x^{3}\right) \\
& +\frac{29}{324000}\left(900 c_{1} x^{3}+1740 c_{1}^{2} x^{3}+841 c_{1}^{3} x^{3}+900 c_{2} x^{3}+1740 c_{1} c_{2} x^{3}+900 c_{3} x^{3}\right) .
\end{aligned}
$$

The approximate solution (49) contain auxiliary constants, the values of constants $c_{1}=-1.35766370, c_{2}=-1.37973022$ and $c_{3}=-0.83053608$ are calculated using method of least squares.

With these constants the approximate solution (49) become

$$
u=-0.0833333 x^{3}+\frac{1}{12}\left(12-12 e^{x}+x^{3}\right) .
$$

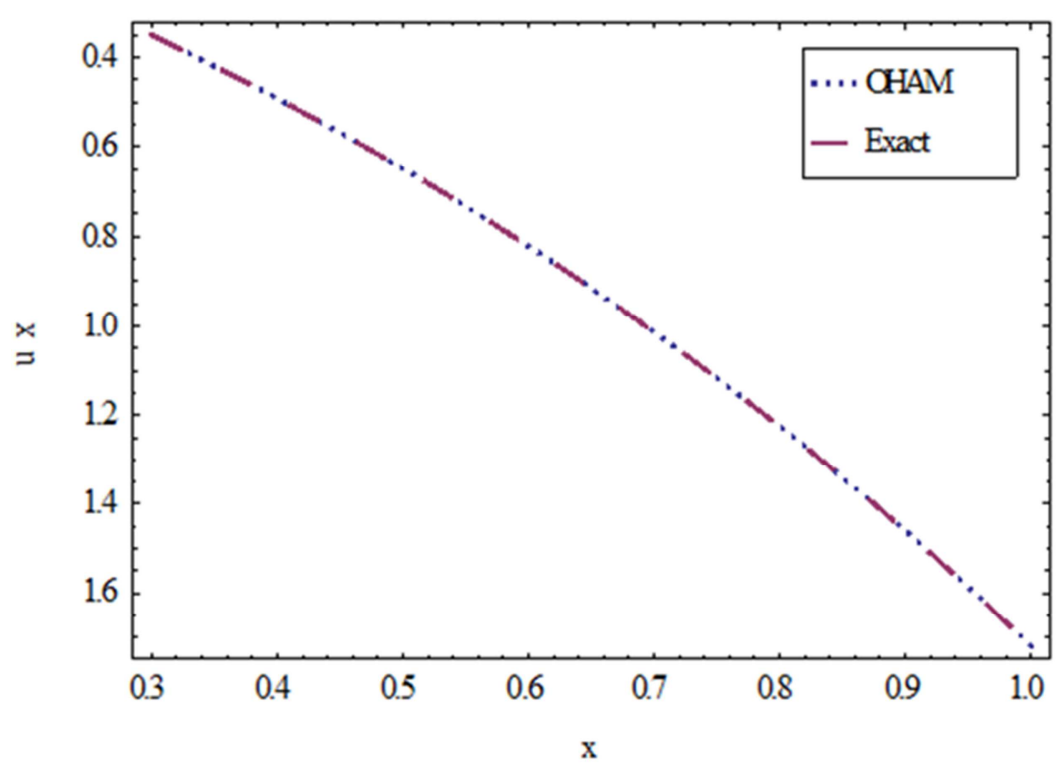

Figure 3. $2 D$ plots of OHAM versus exact solution. 
Table 3. Show the comparison of $3^{\text {rd }}$ order OHAM solution with $4^{\text {rd }}$ order ADM [19] and exact solution.

\begin{tabular}{lllll}
\hline $\mathbf{X}$ & Exact solutions & OHAM solutions & ADM absolute errors & OHAM absolute Errors \\
\hline 0 & 0 & 0 & 0 & 0 \\
0.1 & -0.10517 & -0.10517 & $2.67490 \times 10^{-8}$ & $2.13992 \times 10^{-7}$ \\
0.2 & -0.22140 & -0.22140 & $7.22222 \times 10^{-7}$ & $1.38778 \times 10^{-17}$ \\
0.3 & -0.34985 & -0.34985 & $1.71193 \times 10^{-6}$ & $8.32667 \times 10^{-17}$ \\
0.4 & -0.49182 & -0.49182 & $3.34362 \times 10^{-6}$ & 0 \\
0.5 & -0.64872 & -0.64872 & $5.77778 \times 10^{-6}$ & $1.11022 \times 10^{-17}$ \\
0.6 & -0.82211 & -0.82211 & $9.17490 \times 10^{-6}$ & 0 \\
0.7 & -1.01375 & -1.01375 & $1.36955 \times 10^{-5}$ & $2.22045 \times 10^{-17}$ \\
0.8 & -1.22554 & -1.22554 & $1.95000 \times 10^{-5}$ & 0 \\
0.9 & -1.45960 & -1.45960 & $2.67490 \times 10^{-5}$ & 0 \\
1 & -1.71828 & -1.71828 & & 0 \\
\hline
\end{tabular}

Problem 4

Consider the following Volterra integro-differential equation of the form

$$
u^{\prime \prime}(x)=1+x+\int_{0}^{x}(x-t) u(t) d t, u(0)=1, u^{\prime}(0)=1,
$$

Eq. (51) has exact solution $u(x)=e^{x}$. By using the Basic Idea of OHAM, we have a series of problems

$$
\begin{gathered}
u_{0}{ }^{\prime \prime}(x)=1+x, \quad u_{0}(0)=1, u_{0}{ }^{\prime}(0)=1, \\
u_{1}^{\prime \prime}(x)=-c_{1} \int_{0}^{x}(-t+x) u_{0}(t) d t, \quad u_{1}(0)=0, u_{1}^{\prime}(0)=0, \\
u_{2}^{\prime \prime}(x)=\left(1+c_{1}\right) u_{1}^{\prime \prime}(x)-c_{2} \int_{0}^{x}(-t+x) u_{0}(t) d t-c_{1} \int_{0}^{x}(-t+x) u_{1}(t) d t, u_{2}(0)=0, u_{2}{ }^{\prime}(0)=0, \\
u_{3}^{\prime \prime}(x)=\left(1+c_{1}\right) u_{2}{ }^{\prime \prime}(x)+c_{2} u_{1}^{\prime \prime}(x)-c_{3} \int_{0}^{x}(-t+x) u_{0}(t) d t-c_{2} \int_{0}^{x}(-t+x) u_{1}(t) d t \\
-c_{1} \int_{0}^{x}(-t+x) u_{2}(t) d t, \quad u_{3}(0)=0, u_{3}{ }^{\prime}(0)=0 .
\end{gathered}
$$

Solving eqs. $(52-55)$, we have

$$
\begin{gathered}
u_{0}(x)=\frac{1}{6}\left(6+6 x+3 x^{2}+x^{3}\right), \\
u_{1}(x)=\frac{1}{5040}\left(-210 c_{1} x^{4}-42 c_{1} x^{5}-7 c_{1} x^{6}-c_{1} x^{7}\right), \\
u_{2}(x)=\frac{1}{39916800}\left(-1663200 c_{1} x^{4}-1663200 c_{1}^{2} x^{4}-1663200 c_{2} x^{4}-332640 c_{1} x^{5}\right. \\
-332640 c_{1}^{2} x^{5}-332640 c_{2} x^{5}-55440 c_{1} x^{6}-55440 c_{1}^{2} x^{6}-55440 c_{2} x^{6}-7920 c_{1} x^{7} \\
\left.-7920 c_{1}^{2} x^{7}-7920 c_{2} x^{7}+990 c_{1}^{2} x^{8}+110 c_{1}^{2} x^{9}+11 c_{1}^{2} x^{10}+c_{1}^{2} x^{11}\right),
\end{gathered}
$$




$$
\begin{aligned}
& u_{3}(x)=\frac{1}{1307674368000}\left(-54486432000 c_{1} x^{4}-108972864000 c_{1}^{2} x^{4}\right. \\
& -54486432000 c_{1}^{3} x^{4}-54486432000 c_{2} x^{4}-108972864000 c_{1} c_{2} x^{4}-54486432000 c_{3} x^{4} \\
& -10897286400 c_{1} x^{5}-21794572800 c_{1}^{2} x^{5}-54486432000 c_{3} x^{4}-10897286400 c_{1} x^{5} \\
& -21794572800 c_{1}^{2} x^{5}-10897286400 c_{3} x^{5}-1816214400 c_{1} x^{6}-3632428800 c_{1}^{2} x^{6} \\
& -1816214400 c_{1}^{3} x^{6}-1816214400 c_{2} x^{6}-3632428800 c_{1} c_{2} x^{6}-1816214400 c_{3} x^{6} \\
& -259459200 c_{1} x^{7}-518918400 c_{1}^{2} x^{7}-259459200 c_{1}^{3} x^{7}-259459200 c_{2} x^{7} \\
& -518918400 c_{1} c_{2} x^{7}-259459200 c_{3} x^{7}+64864800 c_{1}^{2} x^{8}+64864800 c_{1}^{3} x^{8} \\
& +64864800 c_{1} c_{2} x^{8}+7207200 c_{1}^{2} x^{9}+7207200 c_{1}^{3} x^{9}+7207200 c_{1} c_{2} x^{9} x^{9} \\
& +720720 c_{1}^{2} x^{10}+720720 c_{1}^{3} x^{10}+720720 c_{1} c_{2} x^{10}+65520 c_{1}^{2} x^{11}+65520 c_{1}^{3} x^{11} \\
& \left.+65520 c_{1} c_{2} x^{11}-2730 c_{1}^{3} x^{12}-210 c_{1}^{3} x^{13}-15 c_{1}^{3} x^{14}-c_{1}^{3} x^{15}\right) .
\end{aligned}
$$

The $3^{\text {rd }}$ order approximate solution by OHAM is $u=u_{0}+u_{1}+u_{2}+u_{3}$

$$
\begin{aligned}
& u=\frac{1}{6}\left(6+6 x+3 x^{2}+x^{3}\right)+\frac{1}{5040}\left(-210 c_{1} x^{4}-42 c_{1} x^{5}-7 c_{1} x^{6}-c_{1} x^{7}\right) \\
& +\frac{1}{39916800}\left(-1663200 c_{1} x^{4}-1663200 c_{1}^{2} x^{4}-1663200 c_{2} x^{4}-332640 c_{1} x^{5}\right. \\
& -332640 c_{1}^{2} x^{5}-332640 c_{2} x^{5}-55440 c_{1} x^{6}-55440 c_{1}^{2} x^{6}-55440 c_{2} x^{6}-7920 c_{1} x^{7} \\
& \left.-7920 c_{1}^{2} x^{7}-7920 c_{2} x^{7}+990 c_{1}^{2} x^{8}+110 c_{1}^{2} x^{9}+11 c_{1}^{2} x^{10}+c_{1}^{2} x^{11}\right) \\
& +\frac{1}{1307674368000}\left(-54486432000 c_{1} x^{4}-108972864000 c_{1}^{2} x^{4}\right. \\
& -54486432000 c_{1}^{3} x^{4}-54486432000 c_{2} x^{4}-108972864000 c_{1} c_{2} x^{4}-54486432000 c_{3} x^{4} \\
& -10897286400 c_{1} x^{5}-21794572800 c_{1}^{2} x^{5}-54486432000 c_{3} x^{4}-10897286400 c_{1} x^{5} \\
& -21794572800 c_{1}^{2} x^{5}-10897286400 c_{3} x^{5}-1816214400 c_{1} x^{6}-3632428800 c_{1}^{2} x^{6} \\
& -1816214400 c_{1}^{3} x^{6}-1816214400 c_{2} x^{6}-3632428800 c_{1} c_{2} x^{6}-1816214400 c_{3} x^{6} \\
& -259459200 c_{1} x^{7}-518918400 c_{1}^{2} x^{7}-259459200 c_{1}^{3} x^{7}-259459200 c_{2} x^{7} \\
& -518918400 c_{1} c_{2} x^{7}-259459200 c_{3} x^{7}+64864800 c_{1}^{2} x^{8}+64864800 c_{1}^{3} x^{8} \\
& +64864800 c_{1} c_{2} x^{8}+7207200 c_{1}^{2} x^{9}+7207200 c_{1}^{3} x^{9}+7207200 c_{1} c_{2} x^{9} x^{9} \\
& +720720 c_{1}^{2} x^{10}+720720 c_{1}^{3} x^{10}+720720 c_{1} c_{2} x^{10}+65520 c_{1}^{2} x^{11}+65520 c_{1}^{3} x^{11} \\
& \left.+65520 c_{1} c_{2} x^{11}-2730 c_{1}^{3} x^{12}-210 c_{1}^{3} x^{13}-15 c_{1}^{3} x^{14}-c_{1}^{3} x^{15}\right) .
\end{aligned}
$$

The approximate solution (59) contain auxiliary constants, the value of constants $c_{1}=-1.0000215, c_{2}=0$ and $c_{3}=0$ are calculated using method of least squares.

With these constants the approximate solution (59) become

$$
\begin{aligned}
u= & \frac{1}{6}\left(6+6 x+3 x^{2}+x^{3}\right)+\frac{1}{5040}\left(210.0045 x^{4}+42.0009 x^{5}+7.0002 x^{6}+1.00002 x^{7}\right) \\
& +\frac{1}{39916800}\left(-35.7551 x^{4}-7.1510 x^{5}-1.1918 x^{6}-0.17026 x^{7}+990.0426 x^{8}\right. \\
& \left.+110.0047 x^{9}+11.0005 x^{10}+1.00004 x^{11}\right)+\frac{1}{1307674368000}\left(25.1806 x^{4}+5.0361 x^{5}\right. \\
& +0.8393 x^{6}+0.1199 x^{7}-1394.4790 x^{8}-154.9421 x^{9}-15.4942 x^{10}-1.4086 x^{11} \\
& \left.+2730.1760 x^{12}+210.0135 x^{13}+15.0009 x^{14}+1.00006 x^{15}\right)
\end{aligned}
$$


Table 4. Show the comparison of $3^{\text {rd }}$ order OHAM solution and exact solution.

\begin{tabular}{llll}
\hline $\mathbf{X}$ & Exact & OHAM & Absolute errors \\
\hline 0 & 1 & 1 & 0 \\
0.1 & 1.10517 & 1.10517 & $4.44089 \times 10^{-16}$ \\
0.2 & 1.22140 & 1.22140 & 0 \\
0.3 & 1.34986 & 1.34986 & 0 \\
0.4 & 1.49182 & 1.49182 & 0 \\
0.5 & 1.64872 & 1.64872 & $2.22045 \times 10^{-16}$ \\
0.6 & 1.82212 & 1.82212 & $4.44089 \times 10^{-16}$ \\
0.7 & 2.01375 & 2.01375 & $4.44089 \times 10^{-16}$ \\
0.8 & 2.22554 & 2.22554 & $2.22045 \times 10^{-15}$ \\
0.9 & 2.45960 & 2.45960 & $1.50990 \times 10^{-14}$ \\
1 & 2.71828 & 2.71828 & $5.68434 \times 10^{-14}$ \\
\hline
\end{tabular}

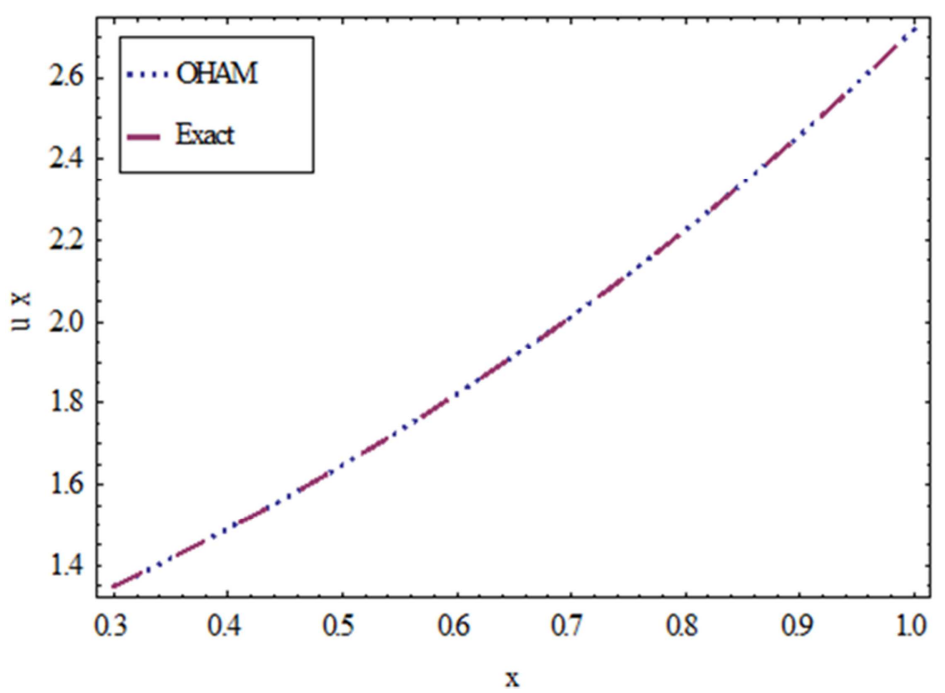

Figure 4. $2 D$ plots of OHAM versus exact solution.

Problem 5

Consider the following Volterra integro-differential equation of the form

$$
u^{\prime}(x)=2+\int_{0}^{x} u(t) d t, \quad u(0)=2
$$

Eq. (61) has exact solution $u(x)=2 e^{x}$. By using the basic idea of OHAM, we have a series of problems

$$
\begin{gathered}
u_{0}{ }^{\prime}(x)=2, \quad u_{0}(0)=2, \\
u_{1}{ }^{\prime}(x)=-c_{1} \int_{0}^{x} u_{0}(t) d t, \quad u_{1}(0)=0, \\
u_{2}{ }^{\prime}(x)=\left(1+c_{1}\right) u_{1}{ }^{\prime}(x)-c_{2} \int_{0}^{x} u_{0}(\mathrm{t}) d t-c_{1} \int_{0}^{x} u_{1}(t) d t, \quad u_{2}(0)=0, \\
u_{3}{ }^{\prime}(x)=\left(1+c_{1}\right) u_{2}{ }^{\prime}(x)+c_{2} u_{1}{ }^{\prime}(x)-c_{1} \int_{0}^{x} u_{2}(t) d t-c_{2} \int_{0}^{x} u_{1}(t) d t-c_{3} \int_{0}^{x} u_{0}(t) d t, \quad u_{3}(0)=0,
\end{gathered}
$$

Solving Eqs. $(62-65)$, we have

$$
u_{0}(x)=2(1+x)
$$




$$
\begin{gathered}
u_{1}(x)=\frac{1}{3}\left(-3 c_{1} x^{2}-c_{1} x^{3}\right), \\
u_{2}(x)=\frac{1}{60}\left(-60 c_{1} x^{2}-60 c_{1}^{2} x^{2}-60 c_{2} x^{2}-20 c_{1} x^{3}-20 c_{1}^{2} x^{3}-20 c_{2} x^{3}+5 c_{1}^{2} x^{4}+c_{1}^{2} x^{5}\right), \\
u_{3}(x)=\frac{1}{2520}\left(-2520 c_{1} x^{2}-5040 c_{1}^{2} x^{2}-2520 c_{1}^{3} x^{2}-2520 c_{2} x^{2}-5040 c_{1} c_{2} x^{2}-2520 c_{3} x^{2}\right. \\
-840 c_{1} x^{3}-1680 c_{1}{ }^{2} x^{3}-840 c_{1}^{3} x^{3}-840 c_{2} x^{3}-1680 c_{1} c_{2} x^{3}-840 c_{3} x^{3}+420 c_{1}{ }^{2} x^{4} \\
\left.+420 c_{1}^{3} x^{4}+420 c_{1} c_{2} x^{4}+84 c_{1}^{2} x^{5}+84 c_{1}^{3} x^{5}+84 c_{1} c_{2} x^{5}-7 c_{1}^{3} x^{6}-c_{1}^{3} x^{7}\right) .
\end{gathered}
$$

The $3^{\text {rd }}$ order approximate solution by OHAM is $u=u_{0}+u_{1}+u_{2}+u_{3}$

$$
\begin{aligned}
u=2(1+ & x)+\frac{1}{3}\left(-3 c_{1} x^{2}-c_{1} x^{3}\right)+\frac{1}{60}\left(-60 c_{1} x^{2}-60 c_{1}^{2} x^{2}-60 c_{2} x^{2}-20 c_{1} x^{3}-20 c_{1}^{2} x^{3}\right. \\
& \left.-20 c_{2} x^{3}+5 c_{1}^{2} x^{4}+c_{1}^{2} x^{5}\right)+\frac{1}{2520}\left(-2520 c_{1} x^{2}-5040 c_{1}^{2} x^{2}-2520 c_{1}^{3} x^{2}-2520 c_{2} x^{2}\right. \\
& -5040 c_{1} c_{2} x^{2}-2520 c_{3} x^{2}-840 c_{1} x^{3}-1680 c_{1}{ }^{2} x^{3}-840 c_{1}^{3} x^{3}-840 c_{2} x^{3}-1680 c_{1} c_{2} x^{3} \\
& -840 c_{3} x^{3}+420 c_{1}{ }^{2} x^{4}+420 c_{1}^{3} x^{4}+420 c_{1} c_{2} x^{4}+84 c_{1}^{2} x^{5}+84 c_{1}^{3} x^{5}+84 c_{1} c_{2} x^{5}-7 c_{1}^{3} x^{6} \\
& \left.-c_{1}^{3} x^{7}\right)
\end{aligned}
$$

The estimate solution (70) contain auxiliary constants, the value of constants $c_{1}=-1.01277013, c_{2}=0.00018746$ and $c_{3}=-0.00000875$ are calculated using method of least squares.

With these constants the approximate solution (70) become

$$
\begin{gathered}
u=2(1+x)+\frac{1}{3}\left(3.0383 x^{2}+1.0128 x^{3}\right)+\frac{1}{60}\left(-0.7872 x^{2}-0.2624 x^{3}+5.1285 x^{4}+1.0257 x^{5}\right) \\
+\frac{1}{2520}\left(0.9227 x^{2}+0.3076 x^{3}-5.5810 x^{4}-1.1162 x^{5}+7.2716 x^{6}+1.0388 x^{7}\right) .
\end{gathered}
$$

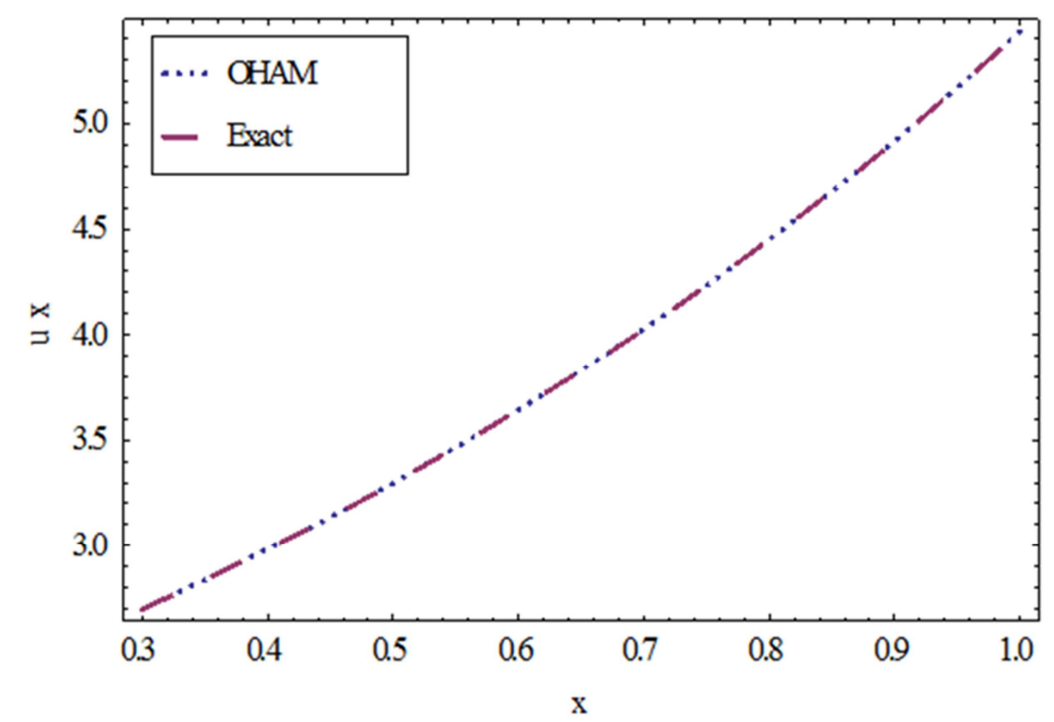

Figure 5. 2D plots of OHAM versus exact solution.

Table 5. Show the comparison of $3^{\text {rd }}$ order OHAM solution and exact solution.

\begin{tabular}{llll}
\hline $\mathbf{X}$ & Exact & OHAM & Absolute errors \\
\hline 0 & 2 & 2 & 0 \\
0.1 & 2.21034 & 2.21034 & $1.54105 \times 10^{-7}$ \\
0.2 & 2.44281 & 2.44281 & $5.52394 \times 10^{-7}$ \\
\hline
\end{tabular}




\begin{tabular}{llll}
\hline $\mathbf{X}$ & Exact & OHAM & Absolute errors \\
\hline 0.3 & 2.69972 & 2.69972 & $1.00035 \times 10^{-7}$ \\
0.4 & 2.98365 & 2.98365 & $1.25368 \times 10^{-6}$ \\
0.5 & 3.29744 & 3.29744 & $1.15329 \times 10^{-6}$ \\
0.6 & 3.64424 & 3.64424 & $7.54767 \times 10^{-7}$ \\
0.7 & 4.02751 & 4.02751 & $3.75290 \times 10^{-7}$ \\
0.8 & 4.45108 & 4.45108 & $4.51330 \times 10^{-7}$ \\
0.9 & 4.91921 & 4.91921 & $1.06711 \times 10^{-7}$ \\
1 & 5.43656 & 5.43656 & $9.77423 \times 10^{-7}$ \\
\hline
\end{tabular}

\section{Results and Discussions}

Table 1 show the comparison of $3^{\text {rd }}$ order OHAM solutions with $5^{\text {th }}$ order ADM solutions for problem 1. Tables $(2-3)$ show the comparison of $3^{\text {rd }}$ order OHAM solutions with $4^{\text {th }}$ order ADM solutions for problem $(2-3)$, respectively. Tables $(4-5)$ show the absolute errors of $3^{\text {rd }}$ order OHAM solution for problems $(4-5)$, respectively. Figures $(1-5)$ show the 2D plots of exact solution versus approximate solution by OHAM for problems $(1-5)$, respectively. The consistency and effectiveness of OHAM has been cleared from all these solved problems.

\section{Conclusion}

The intent of this attempt is to check the ability of OHAM for solving Fredholm type integro-differential equations and Volterra type integro-differential equations. OHAM gave straight forward approximate solution for these integrodifferential eqautions which has close resemblance with the exist solution. From above results and discussions, it is clear that OHAM is more reliable, precise and converges faster to exact solution than ADM. The accuracy of proposed method can further be improved by taking higher order approximations.

Finally, it should be added that the presented technique has the potential to be practical in solving linear and non-linear fractional order integro-differentail equations.

\section{References}

[1] Shang, X., \& Han, D. (2010). Application of the variational iteration method for solving nth-order integro-differential equations. Journal of Computational and Applied Mathematics, 234 (5), 1442-1447.

[2] Wazwaz, A. M. (2014). The variational iteration method for solving the Volterra integro-differential forms of the LaneEmden equations of the first and the second kind. Journal of Mathematical Chemistry, 52 (2), 613-626.

[3] Eshkuvatov, Z. K., Zulkarnain, F. S., Muminov, Z., \& Long, N. M. A. N. (2017). Convergence of modified homotopy perturbation method for Fredholm-Volterra integro-differential equation of order $\mathrm{m}$. Malaysian Journal of Fundamental and Applied Sciences, 13 (4-1), 340-345.

[4] Elbeleze, A. A., Kılıçman, A., \& Taib, B. M. (2016). Modified Homotopy Perturbation Method for Solving Linear Second-
Order Fredholm Integro-Differential Equations. Filomat, 30 (7), 1823-1831.

[5] Shah, k., \& Singh, T. (2015). • Solution of second kind Volterra Integral and Integro-defferential equation by homotopy analysis method. International Journal of Mathematical Archive EISSN 6 (4), 2229-5046.

[6] Mohamed, M. S., Gepreel, K. A., Alharthi, M. R., \& Alotabi, R. A. (2016). Homotopy analysis transform method for integrodifferential equations. General Mathematics Notes, 32 (1), 32-48.

[7] Abbas, Z., Vahdati, S., Ismail, F., \& Dizicheh, A. K. (2010). Application of homotopy analysis method for linear integrodifferential equations. In International Mathematical Forum. 5 (5), 237-249.

[8] Saeidy, M., Matinfar, M., \& Vahidi, J. (2010). Analytical solution of BVPs for fourth-order integro-differential equations by using homotopy analysis method. International Journal of Nonlinear Science, 9 (4), 414-421.

[9] Aruchunan, E., \& Sulaiman, J. (2011). Half-sweep conjugate gradient method for solving first order linear Fredholm integro-differential equations. Australian Journal of Basic and Applied Sciences, 5 (3), 38-43.

[10] Alao, S., Akinboro, F. S., Akinpelu, F. O., \& Oderinu, R. A. (2014). Numerical Solution of Integro-Differential Equation using Adomian Decomposition and Variational Iteration Methods. IOSR Journal of Mathematics, 10 (4), 18-22.

[11] Vahidi, A. R., Babolian, E., Cordshooli, G. A., \& Azimzadeh, Z. (2009). Numerical solution of Fredholm integro-differential equation by Adomian's decomposition method. International Journal of Mathematical Analysis, 3 (36), 1769-1773.

[12] Rahmani, L., Rahimi, B., \& Mordad, M. (2011). Numerical Solution of Volterra-Fredholm Integro-Differential Equation by Block Pulse Functions and Operational Matrices. General Mathematics Note, 4 (2), 37-48.

[13] Marinca, V., \& Herişanu, N. (2008). Application of optimal homotopy asymptotic method for solving nonlinear equations arising in heat transfer. International Communications in Heat and Mass Transfer, 35 (6), 710-715.

[14] Herişanu, N., Marinca, V., Dordea, T., \& Madescu, G. (2008). A new analytical approach to nonlinear vibration of an electrical machine. Proceedings of the Romanian AcademySeries A, 9 (3), 229-236.

[15] Khan, N., Hashmi, M. S., Iqbal, S., \& Mahmood, T. (2014). Optimal homotopy asymptotic method for solving Volterra integral equation of first kind. Alexandria Engineering Journal, 53 (3), 751-755.

[16] Almousa, M., \& Ismail, A. (2013). Optimal homotopy asymptotic method for solving the linear Fredholm integral equations of the first kind. Abstract and Applied Analysis, 1-6. 
[17] Hashmia, M. S., Khanb, N., Iqbalc, S., \& Zahida, M. A. (2016). Exact solution of Fredholmintegro-differential equations using optimal homotopy asymptotic method. Journal of. Applied Environmental and Biological Sciences, 6 (4S), 162-166.

[18] Du Han, Y., \& Yun, J. H. (2013). Optimal homotopy asymptotic method for solving integro-differential equations. International Journal of Applied Mathematics, 43 (3).

[19] Karim, M. F., Mohamad, M., Rusiman, M. S., Che-Him, N., Roslan, R., \& Khalid, K. (2018) ADM For Solving Linear Second-Order Fredholm Integro-Differential Equations. Journal of Physics, 995 (1), 1-10. 